\title{
Geometrical structure effect on localization length of carbon nanotubes
}

\author{
Wen-Qi Ran ${ }^{1} \ddagger$, Jun Chang ${ }^{2}$, Han-Tao Lu ${ }^{1}$, Yue-Hua $\mathrm{Su}^{3}$, \\ Hong-Gang $\mathrm{Luo}^{2}$ and Tao Xiang ${ }^{2}$ \\ ${ }^{1}$ School of Physics, Peking University, Beijing 100871, China \\ ${ }^{2}$ Institute of Theoretical Physics and Interdisciplinary Center of Theoretical Studies, \\ Chinese Academy of Sciences, P.O. Box 2735, Beijing 100080, China \\ ${ }^{3}$ Center for Advanced Study, Tsinghua University, Beijing 100084, China
}

\begin{abstract}
The localization length and density of states of carbon nanotubes are evaluated within the tight-binding approximation. By comparison with the corresponding results for the square lattice tubes, it is found that the hexagonal structure affects strongly the behaviors of the density of states and localization lengths of carbon nanotubes.
\end{abstract}

PACS numbers: 73.63.Fg, 61.43.Bn, 73.61.Wp

‡ wenqi.ran@gmail.com 
In recent years, there have been extensive interests in the study of carbon nanotubes' transport properties. [1, 2, 3, 4] In particular, extremely high or even ballistic conductance was reported in carbon nanotubes. [5] 6] This indicates that carbon nanotubes can be synthesized with high purity. However, as there are always some defects or dislocations inside the tubes or caused by the substrate or attached to the tubes, much attention has been paid to the study of the disorder effect in carbon nanotubes. [7, 8] In 1992, Minitmire 9] predicted that a carbon nanotube can behave as a metal or a semiconductor depending on its chirality. This prediction was later confirmed by scanning tunneling microscopic measurements. [10] White argued that due to the $C_{N}$ rotation symmetry the scattering of electrons is significantly reduced by the doughnut-like wave packet confined along the tube but extended around its circumference in the $(N, N)$ tubes. [11] Furthermore, it is believed that semiconducting tubes are more sensitive to long-range disorder than metallic tubes. [12, 13]

In this letter we explore the effect of geometrical structure on the scattering of electrons in carbon nanotubes. We evaluate the localization length as well as the density of states with the Green's function method. By comparison with the corresponding quantities for the square lattice tubes, we find that the hexagonal structure can significantly reduce the scattering of electrons by on-site random potentials. The influence of the density of states (DOS) on the localization lengths of carbon nanotubes is also discussed.

Let us start with the Anderson model of random potentials for carbon nanotubes 14

$$
H=\sum_{i} \varepsilon_{i}|i\rangle\left\langle i\left|+\sum_{\langle i j\rangle} t\right| i\right\rangle\langle j|
$$

where $\langle i j\rangle$ means that $i$ and $j$ are nearest neighbors; $\varepsilon_{i}$ is a random on-site potential. It takes any value between $-W$ and $W$ with equal probability. Without loss of generality, we set $t=1$.

Carbon nanotubes can generally be classified by two integers and labeled as $(m, n)$. Two types of carbon nanotubes are of particular interest. One is the armchair type with $m=n$, and the other is the zigzag type with $n=0$. An undoped armchair nanotube is always a metal, but a zigzag nanotube is a metal only when $m=3 k$ with $k$ an integer. In general, it shows that a carbon nanotube is a metal if $m-n$ is a multiple of 3 or a semiconductor otherwise. For simplicity, only the armchair or zigzag nanotubes will be considered below.

A carbon nanotube can be taken as a one dimensional stripe extending along the tube direction. The width of the stripe depends on the structure of the nanotube. For a $(m, 0)$ zigzag tube, the width is $m$. If we use $|l\rangle$ to represent the basis state for the $l$ th slice of the stripe along the tube direction, then the Schrödinger equation for the single-particle eigenfunction, $|\psi\rangle=\sum_{l} a_{l}|l\rangle$, can be determined by the following recursion formula,

$$
V_{l, l+1} a_{l+1}=\left(E-H_{l}\right) a_{l}-V_{l, l-1} a_{l-1},
$$




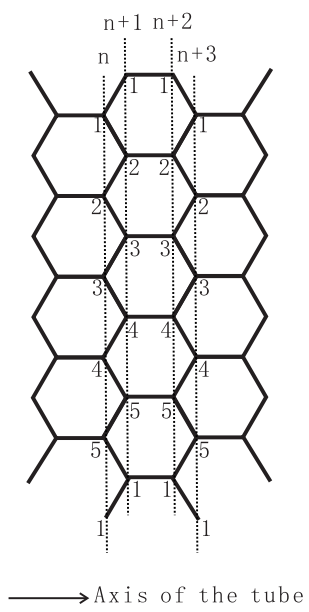

Figure 1. The structure of a unwrapped zigzag $(5,0)$ nanotube. Each slice perpendicular to the tube axis contains five sites connected by the dotted lines.

where $V_{l, l+1}$ is the interaction matrix between two neighboring slices. $H_{l}$ is a diagonal matrix, formed by the random on-site potential $\varepsilon_{i}$ in the $l$ th slice. For square lattices, $V_{l, l+1}$ is simply a unit matrix. However, for carbon nanotubes, it takes a more complicated form. For a zigzag tube, $V_{l, l+1}$ is a simple periodic function of $l$ and the periodicity is 4 . For example, for the $(5,0)$ zigzag nanotube (Fig. 1), $V_{l, l+1}$ are

$$
\begin{aligned}
V_{4 l, 4 l+1} & =\left(\begin{array}{ccccc}
1 & 1 & 0 & 0 & 0 \\
0 & 1 & 1 & 0 & 0 \\
0 & 0 & 1 & 1 & 0 \\
0 & 0 & 0 & 1 & 1 \\
1 & 0 & 0 & 0 & 1
\end{array}\right), \\
V_{4 l+1,4 l+2} & =V_{4 l+3,4 l+4}=I_{5 \times 5}, \\
V_{4 l+2,4 l+3} & =\left(\begin{array}{ccccc}
1 & 0 & 0 & 0 & 1 \\
1 & 1 & 0 & 0 & 0 \\
0 & 1 & 1 & 0 & 0 \\
0 & 0 & 1 & 1 & 0 \\
0 & 0 & 0 & 1 & 1
\end{array}\right),
\end{aligned}
$$

where $I_{5 \times 5}$ is a $5 \times 5$ unit matrix. For other zigzag or armchair tubes, $V_{l, l+1}$ can be similarly defined.

The localization length $\lambda$ is a characteristic length scale for describing the decay of an eigenfunction in space. It is defined by [15]

$$
\frac{1}{\lambda}=-\lim _{l \rightarrow \infty} \frac{1}{2(l-1)} \ln \operatorname{Tr}|\langle 1|G(l)| l\rangle|^{2},
$$

where $\langle 1|G(l)| l\rangle$ denotes the matrix elements of the resolvent $\left(E-H_{l}\right)^{-1}$ between the site states in the first and $l$ th slices. Given a configuration of random potentials, $\lambda$ can 
be evaluated by solving Eq. (2) with the Green's Function approach introduced in Ref. [16].

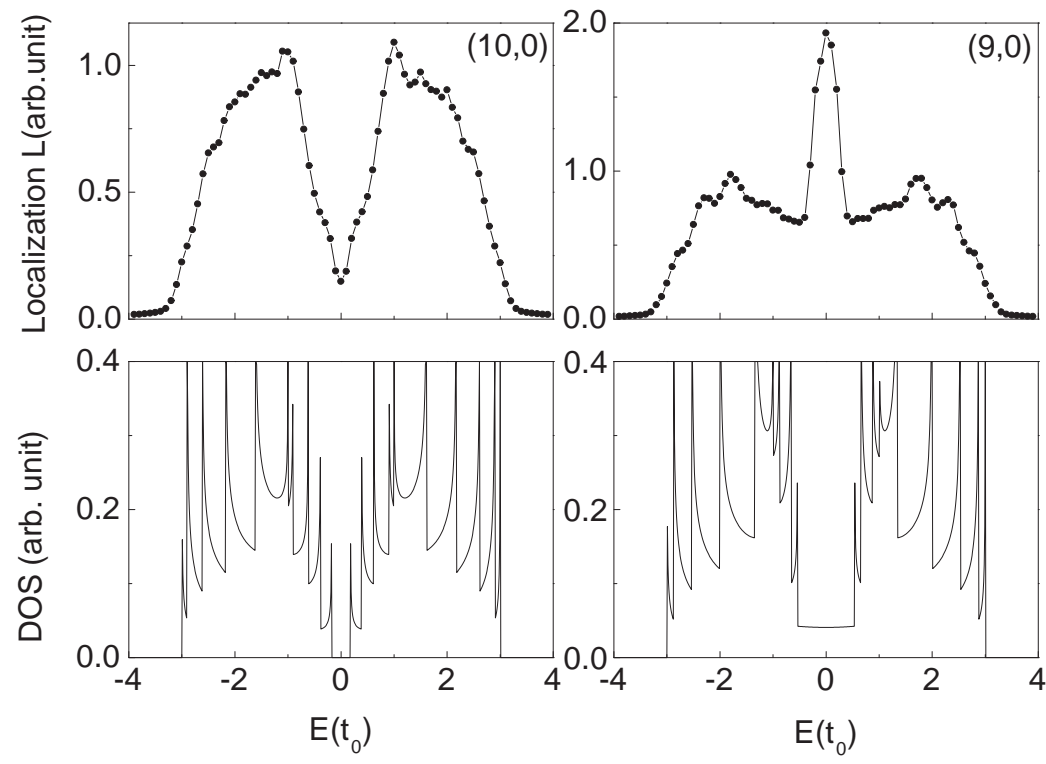

Figure 2. The localization length and the DOS without disorder as a function of energy for the $(10,0)$ and $(9,0)$ carbon nanotubes. The random potential is uniformly distributed within $[-1,1]$.

Figure 2 shows the localization length as a function of energy $E$ for two zigzag nanotubes. The DOS for the corresponding systems without impurities is also shown. For the semiconducting $(10,0)$ zigzag nanotube, $\lambda$ shows a dip at zero energy. This suppression of $\lambda$ is apparently due to the vanishing DOS at $E=0$ since there is a finite energy gap for the $(10,0)$ nanotube at half filling. However, for the $(9,0)$ zigzag nanotube, the DOS is finite and $\lambda$ shows a peak at $E=0$. This shows that the localization length behaves very differently in different type of nanotubes.

The appearance of the zero energy peak of $\lambda$ is a unique property of metallic carbon nanotubes. As shown later, it does not appear in square lattice tubes. For the metallic nanotubes, it is known that both the DOS and the energy separation between the two DOS peaks below and above $E=0$ decrease linearly with increasing tube diameters. However, as shown in Fig. 3, the $E=0$ peak of $\lambda$ is found to increase monotonically with increasing tube diameters. This indicates that the peak of $\lambda$ is anti-correlated with the DOS at $E=0$ for the metallic nanotubes.

In both $(9,0)$ and $(10,0)$ nanotubes, the DOS without impurities shows many diverging peaks at band edges. However, these singularities are smeared out by disorders and $\lambda$ only exhibits some small fluctuation at the energies corresponding to these singularities (Fig. 2).

The difference of $\lambda$ in the semiconducting and metallic nanotubes can be understood 


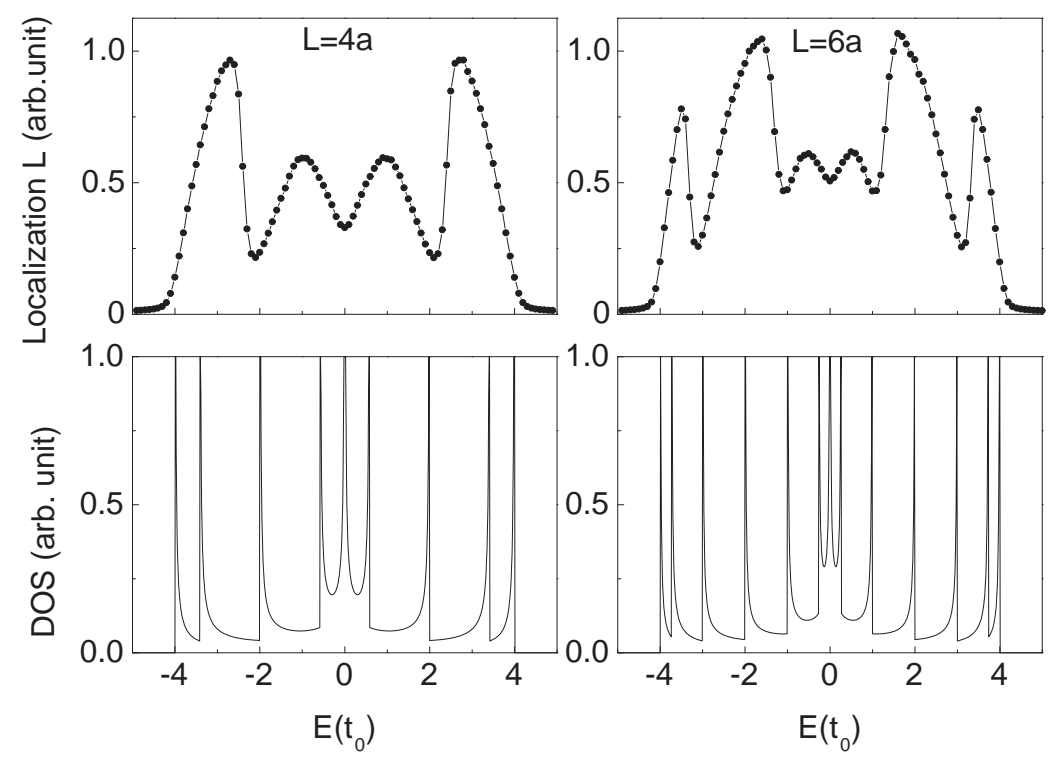

Figure 3. The localization length and the DOS without impurities as a function of energy for square lattice nanotubes with the circumference of $4 \mathrm{a}$ and $6 \mathrm{a}$ ( $\mathrm{a}$ is the lattice constant), respectively. The random potential is uniformly distributed within $[-1,1]$.

from the difference in their DOS. In a disorder system, within the relaxation time approximation, the mean free path $l_{e}$ is related to the DOS by the relation, [17]

$$
l_{e} \simeq \frac{\hbar v_{F}}{2 \pi W^{2} \rho\left(\omega_{F}\right)}
$$

where $v_{F}$ is the Fermi velocity and $\rho\left(\omega_{F}\right)$ is the DOS at the Fermi level. In the weak scattering limit, the velocity of carriers decreases rapidly when the Fermi level shift to the edge of forbidden band, but the DOS tends to diverge because of the presence of van Hove singularities. Thus the mean free path is very short. However, for metallic nanotubes there is a flat band around $E=0$ and the DOS is small but non-zero, the corresponding mean free path should be much larger than the semiconducting case. Since the localization length is roughly proportional to the mean free path $\lambda \propto N_{C} l_{e}$, where $N_{C}$ is the number of channels [11, it is expected the localization length of metallic nanotubes to be much longer than that of semiconducting one in the middle of the band. In additional, the DOS near the Fermi level depend only on the diameter of a metallic nanotube, independent on its chirality. The localization length of a chiral nanotube behaves similarly as for a zigzag or armchair nanotube with close diameter. 4. Thus the above conclusion can be also applied to a chiral nanotube.

For comparison, we have also evaluated the localization length and the corresponding DOS of pure systems for the square lattice nanotubes. As shown in Fig. 3, the DOS in the square lattice tubes diverges at $E=0$. The impurity scattering will suppress the divergence of DOS. However, at the Fermi level, the DOS is still very 
high. Since the mean free path is inversely proportional to the DOS, $l_{e}$ and $\lambda$ of square lattice nanotube are expected to be much shorter than that of carbon nanotube as shown in Fig. 4. Unlike the metallic carbon nanotubes, $\lambda$ in a square lattice nanotube shows a dip around $E=0$. This suggests that $\lambda$ is strongly affected by lattice structures and the hexagonal symmetry of carbon nanotubes can enhance the mobility of the state at the middle of the band.
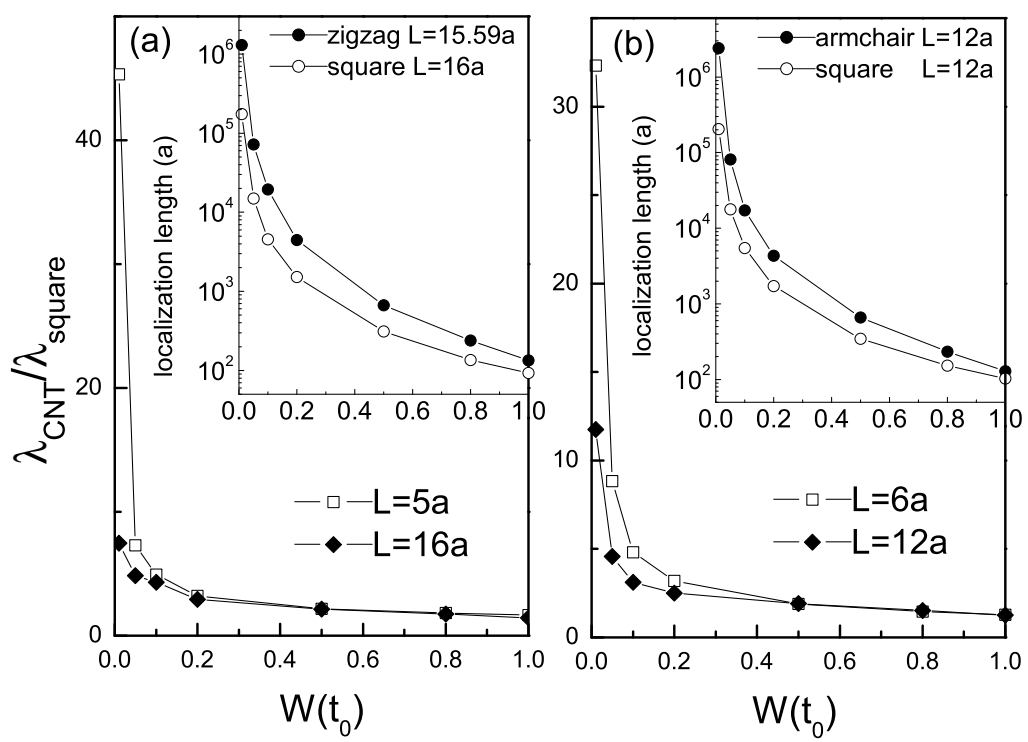

Figure 4. The ratio between the localization length of a metallic carbon nanotube and that of a square lattice nanotube with similar circumference at $E=0$ as a function of the range of the random potential $W$. Left panel: the circumferences of the $(9,0)$ and $(3,0)$ zigzag nanotubes are $15.59 a$ and $5.20 a$, respectively. The corresponding circumferences of the square lattice nanotubes used for comparison are $16 a$ and $5 a$, respectively. The inset compares the localization length for the $(9,0)$ zigzag tube with that of the square lattice tube with the circumference of $16 a$. Right panel: the circumferences of the $(4,4)$ and $(2,2)$ armchair nanotubes are $12 a$ and $6 a$, respectively. The square lattice nanotubes used for comparison have the same circumferences. The inset compares the localization length for the $(4,4)$ armchair tube with that of the square lattice tube with the same circumference.

To further elucidate the difference between carbon and square-lattice nanotubes, we evaluate the ratio between the localization length of metallic carbon nanotubes and that of square nanotubes with close circumference. As shown in Fig. 4, the difference between the localization lengths in these two systems is more apparent when the circumference of nanotubes or the strength of disorder becomes smaller. The localization length of the $(2,2)$ armchair nanotube (Fig. 4b) is more than a order of magnitude larger than the square-lattice one when $W<0.5 t$. This agrees with the result of White and Todorov. [1] It indicates that the mobility of carbon nanotubes is much better than that of 
square lattice ones, especially for thin tubes.

In summary, we have evaluated the localization length and DOS of carbon nanotubes and they are compared with the corresponding quantities for square lattice nanotubes. Our results indicates that in the same strength of disorders, electrons are more extended in a metallic carbon nanotube than in a nanotube rolled from a square lattice, especially in the limit of small diameters. Thus high conductance is favored by the hexagonal structure of carbon nanotubes.

This work has been supported by the National Natural Science Foundation of China under grant No 90203006.

\section{References}

[1] Iijima S 1991 Nature 35456

[2] Ebbesen T W and Ajayan 1992 Nature 358220

[3] Ebbesen T W, Lezec H J, Hiura H, Bennett J W, Ghaemi H F and Thio T 1996 Nature 38254

[4] Yang HT, Dong JM and Xing DY 2001 Chin. Phys. Lett. 181105

[5] Frank S, Poncharal P, Wang Z L and de Heer Walt A 1998 Science 2801744

[6] Lee R S, Kim H J, Fischer J E, Thess A and Smalley R E 1997 Nature 388255

[7] Carroll D L, Redlich Ph, Blase X, Charlier J-C, Curran S, Ajayan P M, Roth S and Rühle M 1998 Phys. Rev. Lett. 812332

[8] Czrew R, Terrones M, Charlier J-C and Blase X et al. 2001 Nano. Lett. 1 457-460

[9] Mintmire J W, Dunlap B I and White C T 1992 Phys. Rev. Lett. 68631

[10] Odom Teri Wang, Huang Jin-Lin, Kim Philip and Lieber Charles M 1998 Nature 39162

[11] White C T and Todorov T N 1998 Nature 393240

[12] McEuen Paul L, Bockrath Marc, Cobden David H, Yoon Young-Gui and Louie Steven G 1999 Phys. Rev. Lett. 835098

[13] Hjort M and Stafstrom S 2001 Phys. Rev. B 63113406

[14] Anderson P W 1958 Phys. Rev. 1091492

[15] MacKinnon A and Kramer B 1981 Phys. Rev. Lett. 471546

[16] MacKinnon A and Kramer B 1983 Z. Phys. B 53 1-13

[17] Roche S, Dresselhaus G, Dresselhaus M S and Saito R 2000 Phys. Rev. B 6216092 